\title{
Specific expression of lactate dehydrogenases in glioblastoma controls intercellular lactate transfer to promote tumor growth and invasion
}

Joris Guyon

INSERM U1029 https://orcid.org/0000-0001-6692-2890

Ignacio Fernandez-Moncada

INSERM U1215

Claire Larrieu

CNRS UMR5095

Cyrielle Bouchez

CNRS UMR5095

Tiffanie Chouleur

INSERM U1029

Heidi Espedal

University of Bergen https://orcid.org/0000-0002-0190-8999

Gro Røsland

University of Bergen

Boutaina Daher

CNRS UMR5095

Aurelien Barre

Univ . Bordeaux, Centre de Bioinformatique de Bordeaux (CBiB)

Benjamin Dartigues

Université de Bordeaux

Aurelien Coffe

Bordeaux Bioinformatic Center CBiB

Justine Rudewicz

Bordeaux Bioinformatic Center CBiB

Irati Romero-Garmendia

CNRS UMR5095

\section{Barbara Klink}

Laboratoire national de santé (LNS) https:// orcid.org/0000-0002-8753-8238

\section{Konrad Grützmann}

Nationales Centrum für Tumorerkrankungen

Marie-Alix Derieppe 
Inserm

\section{Thibaut Molinié}

CNRS UMR5095

\section{Nina Obad}

Department of Biomedecine

\section{Céline Leon}

INSERM and University Bordeaux

\section{Hrvoje Miletic}

Haukeland University Hospital

\section{Giovanni Marsicano}

Inserm https://orcid.org/0000-0003-3804-1951

\section{Macha Nikolski}

Univ . Bordeaux, Centre de Bioinformatique de Bordeaux (CBiB),Univ . Bordeaux, CNRS, IBGC, UMR 5095 https://orcid.org/0000-0001-6059-577X

\section{Rolf Bjerkvig}

University of Bergen https://orcid.org/0000-0002-9622-7263

\section{Andreas Bikfalvi}

University of Bordeaux https://orcid.org/0000-0003-4138-5229

Thomas DAUBON ( $\sim$ thomas.daubon@u-bordeaux.fr)

CNRS UMR5095 https://orcid.org/0000-0002-0319-7617

\section{Article}

Keywords: Glioblastoma, lactate dehydrogenases, energy metabolism, invasion, antiepileptic drug

Posted Date: July 9th, 2021

DOI: https://doi.org/10.21203/rs.3.rs-690811/v1

License: (c) (i) This work is licensed under a Creative Commons Attribution 4.0 International License. Read Full License 


\section{Abstract}

Lactate is a central metabolite in brain physiology, involved in the astrocyte-neuron lactate shuttle, but also contributes to tumor development. Glioblastoma (GBM) is the most common and malignant primary brain tumor in adults, recognized by angiogenic and invasive growth, in addition to its altered metabolism. By adapting their glycolytic or oxidative metabolism, GBM stem-like cells are able to resist chemo- and radiotherapy. We show herein that lactate fuels GBM anaplerosis by replenishing the TCA cycle in absence of glucose. Lactate dehydrogenases (LDH) catalyze the interconversion of pyruvate and lactate. Deletion of either LDHA or LDHB did not alter significantly GBM growth and invasion. However, ablation of both LDH isoforms led to a reduction of tumor growth, and, consequently, to an increase in mouse survival. Comparative transcriptomics and metabolomics revealed metabolic rewiring involving high oxidative phosphorylation (OxPhos) in the double LDHA/B KO group which sensitized tumors to cranial irradiation, massively improving mouse survival. Survival was also increased when control mice were treated with the antiangiogenic treatment, bevacizumab, and the antiepileptic drug, stiripentol which targets LDH activity.

Taken together, this highlights the complex metabolic network in which both LDH A and B are integrated and underscores that combined inhibition of LDHA and B is necessary to impact tumor development. Targeting of these enzymes in combination with anti-angiogenic and repurposed drugs may be of therapeutic benefit, especially when associated with radiotherapy.

\section{Introduction}

GBM is the most common malignant primary brain tumor in adults. Major hallmarks of GBM are high proliferation rate, pronounced angiogenesis, and local invasion ${ }^{1}$. GBM is a highly heterogeneous tumor that exhibit astrocytic-, oligodendrocytic-, neural progenitor-, and mesenchymal features ${ }^{2}$. The current treatment of GBM consists in surgical tumor resection, followed by concomitant radio-chemotherapy. However, this strategy invariably leads to tumor recurrence due the inability to fully eradicate invasive cells and results in poor prognosis ${ }^{3}$. This highlights the need for alternative therapeutic targets to improve this tumor management and treatment. Glioblastoma invasion impacts three types of brain structures: blood vessels, white matter tracts, and the interstitial space 4 . Expression of the hypoxia-inducible factor 1a (HIF1a) expression induced by hypoxia or following anti-angiogenic treatment, significantly impacts GBM invasion ${ }^{5}$ and metabolism ${ }^{6}$. More recently, a connection between neurons and GBM cells expressing $\mathrm{N}$-methyl-D-aspartate receptor (NMDAR) has been reported to sustain GBM invasion?

Unlike non-neoplastic cells that rely on mitochondrial oxidative phosphorylation (also known as "mitochondrial respiration") to produce energy, a common property of many solid cancers, enhanced glucose metabolism ${ }^{8}$. Even under aerobic condition, tumor cells have a high glycolytic rate and production of lactate 9 . This particularity, known as the Warburg effect, is an efficient way to increase biomass production for maintaining a high proliferation rate, and to facilitate invasion by 
microenvironment acidosis ${ }^{8,10}$. Production of lactate, an end product of glycolysis, is catalysed by lactate dehydrogenase (LDH), a tetrameric enzyme composed by two subunits, LDHA and/or LDHB, both encoded by separate genes. Depending on the lactate/pyruvate flux, LDHA and LDHB can both catalyse the conversion of pyruvate into lactate or its retroconversion, both reactions coupled to oxidoreduction of $\mathrm{NAD}^{+} / \mathrm{NADH}$. This leads to increased lactate production (for LDHA) and consumption (for LDHB). Lactate and protons are then extruded from cells by monocarboxylate transporters (MCTs).

Many cancers display high LDHA levels ${ }^{11}$, which is associated with poor patient survival ${ }^{12,13}$. Several preclinical studies have demonstrated a that LDHA inhibition possess anti-proliferative effects ${ }^{14,15}$. One proposed explanation is that LDHA inhibition induces mitochondrial reactive oxygen species (ROS)

production and oxidative damage ${ }^{14,16,17}$. Moreover, several studies have shown that LDHA activity and lactate secretion promote invasion and metastasis ${ }^{18,19}$. In GBM, a relationship between lactate and in vitro migration has also been established ${ }^{20-22}$. LDHA has been found at GBM invasive borders of antiangiogenic-treated tumors ${ }^{6}$. Isocitrate-dehydrogenase (IDH)-mutated gliomas, which are of better prognosis than IDH1wt gliomas, express lower levels of LDHA ${ }^{23}$. Of note, LDHB has not yet been explored in the GBM context, unlike in uterine cancer where LDHB activity modulates autophagy ${ }^{24}$. However, no study to date has examined the role of both LDHA/LDHB in promoting GBM development and invasion.

We therefore aimed to study the impact of LDHA and LDHB activity in GBM development using patientderived GBM stem-like cells cultured as spheroids to maintain parental DNA genotype and tumor phenotype while implanted into rodents ${ }^{25}$.

We show herein that GBM stem-like cells starved from carbon sources and fed with lactate can compensate for the absence of glucose to sustain the TCA cycle to drive proliferation and invasion. Single CRISPR-cas9 LDHA or LDHB knock-out have limited effects on glioblastoma development, but major biological effects are observed in double LDHA/B KO cells. Even under low $\mathrm{O}_{2}$ concentration, double LDHA/B KO cells still rely on TCA and mitochondrial respiration to support tumor growth, which could be further inhibited by irradiation. Finally, treatment with stiripentol (Diacomit $\AA$, Biocodex), a known antiepileptic drug that also block LDH activity ${ }^{26}$, inhibits lactate production and decreases GBM invasion and growth. Collectively, these results demonstrate a central role of LDHA/LDHB and lactate production to support energy metabolism which has a major impact on GBM invasion and growth.

\section{Results}

\section{Expression of LDHA and LDHB are mainly restricted to specific glioblastoma areas}

We have previously showed that regional expression of specific molecules define GBM hallmarks ${ }^{5,27}$. Regional expression of the lactate dehydrogenase isoforms was first analyzed in a patient-derived xenograft mouse model by immunohistochemistry. Both LDH types were predominantly express on invasive cells compared to healthy tissue, however LDHA was mainly expressed in the central hypoxic 
area of the tumor and in some invasive cells, while LDHB was mainly expressed in peripheral tumor areas (Fig 1a). LDHA and LDHB did not colocalize in tumor cells, showing a distinct spatially-restricted expression pattern (Fig 1a). Next, we embedded spheroids invading in a 3D collagen matrix in paraffin, a model that recapitulates GBM oxygenation regional heterogeneity ${ }^{28}$, to analyse LDHA and LDHB expression in coronal paraffin sections (Fig 1b). LDHA was found highly expressed in the central hypoxic area but also in some single invasive cells, while LDHB was highly expressed at spheroid borders and invasive areas (Fig 1C). Of note, only few cells expressed both enzymes. In silico analysis on a single-cell RNAseq database from invasive and central GBM areas ${ }^{29}$ demonstrated a similar pattern, with preferential expression of $\angle D H A$ in the central area and $\angle D H B$ in the peripheral area albeit mRNA of both enzymes were found in a portion of invasive tumor cells (Supplementary Fig 1a). When using data extracted from the IVYGAP project, distinct regional $L D H A$ and $\angle D H B$ expression was observed, as well as a positive correlation between LDHA and the hypoxic transcription factor HIF1a (Supplementary Fig 1b). When analyzed in the GBM-TCGA data set, LDHA was found to be a marker of poor prognosis. LDHB expression was, on the contrary, linked to favourable prognosis (Supplementary Fig 1c). In summary, LDHA and LDHB expression is reciprocally restricted to hypoxic or peripheral areas.

\section{Lactate modulates glioblastoma invasion by fuelling energy metabolism pathways}

On physiological conditions, brain tissue is exposed to low level of oxygen, i.e. physiological hypoxia, that ranges from 0.5 to $7 \%$ depending on the distance from blood vessels ${ }^{30}$. Local $\mathrm{O}_{2}$ concentration can be even lower in brain tumors, reaching around $0.1 \%$ in the core area. We, therefore, investigated whether stem-like cells (P3 or BL13 cells) in culture express LDHA and/or LDHB, and whether expression of these enzymes can be modified by incubating cells at $0.1 \% \mathrm{O}_{2}$. We observed that LDHA expression was upregulated under hypoxic conditions ( $3 x$ fold induction at 72 hours), while LDHB expression did not change (Fig 2a and Supplementary Fig 2a). Lactate production was also increased under hypoxia 0.1\%, correlating with higher LDH activity in P3 and in BL13 cells (Fig $\mathbf{2 b - d}$ and Supplementary Fig $\mathbf{2 b - d}$ ).

Lactate has been reported to sustain tumor growth via monocarboxylate transporters (MCT1/4) ${ }^{31}$. To this aim, we tested the effect of lactate on tumor cell proliferation and invasion. To rule out a lactate-induced cytotoxic effect, lactate was added to the medium of P3 cells and cytotoxicity was measured. Lactate concentrations starting at $30 \mathrm{mM}$ induced cytotoxicity at 24 hours (Supplementary Fig 2e). The effect of non-cytotoxic lactate concentrations was then tested and spheroid growth and invasion measured. Spheroid growth was significantly inhibited by $20 \mathrm{mM}$ lactate (Fig 2e), while cell invasion increased in the presence of lactate from $10 \mathrm{mM}$ onwards (Fig 2f). Lactate treatment also induced changes in cell morphology, promoting an elongated cell shape, reminiscent of a mesenchymal phenotype (Supplementary Fig 2f). In absence of glucose, lactate by itself induced a strong effect on cell invasion (Fig $\mathbf{2 g}$ ). To rule out an effect of acidification, we tested cell invasion at the same $\mathrm{pH}$ of lactate treatment ( $\mathrm{pH}$ 6.8), and no effect was observed (Fig $\mathbf{2 g}$ ). Rotenone, a respiratory chain complex I inhibitor, completely blocked lactate-induced invasion. This suggests that lactate is fuelling mitochondria activity 
to promote invasion (Fig $\mathbf{2 g}$ ). Pyruvate, the main LDH substrate was then tested in invasion experiments, and invasion rates was increased in comparison to control conditions but remained lower than under lactate stimulation (Supplementary Fig $\mathbf{2 g}$ ). Of note, the respiration capacity of P3 cells was similar in complete medium, with glucose or lactate (Fig $\mathbf{2 h}$ ). Lactate consumption by GBM mitochondrial activity was followed by $\left[{ }^{13} \mathrm{C}_{3}\right]$ lactate infusion in glucose-starved $\mathrm{P} 3$ cells, wherein labelled TCA intermediates (citrate, oxoglutarate) and amino acids (alanine, glutamate, glutamine) were detected in the endometabolome after 1 hour but also in the exometabolome (Fig $2 \mathbf{i}$ and Supplementary Fig 3ac). Intracellular abundance of lactate and pyruvate was found unchanged along the experiment (Figure $2 \mathbf{i}$ and Supplementary Figure $3 a$ ), but the amount of $\left[{ }^{13} \mathrm{C}_{3}\right]$ lactate and $\left[{ }^{13} \mathrm{C}_{3}\right]$ pyruvate immediately reached its maximum level (Supplementary Figure $3 b$ ). The appearance of $\left[{ }^{13} \mathrm{C}_{2}\right]$ citrate from the beginning indicated a fast reaction from acetyl-CoA, and higher isotopologues increased quickly after, indicating an active TCA cycle (Supplementary Figure $3 c$ ). These results shed light on the pathways involved in lactate metabolism of GBM cells and is a direct demonstration that lactate is used as an energy source via the TCA cycle. Only a slight increase or almost no labelling of amino-acids not associated with the TCA cycle or gluconeogenesis was observed and, thus, this was not included in the analysis.

\section{Double LDHAVB KO impairs in vitro and in vivo GBM growth and invasion}

First, we transduced P3 and BL13 cells with lentiviral Crispr-Cas9 constructs to fully eliminate LDHA and/or LDHB expressions (sgControl, sgLDHA, sgLDHB, and sgLDHA/B). Knock-out (KO) was validated by Western-blot blot in single LDHA or LDHB KO cells, and in double LDHA/B KO cells at 21 or $0.1 \% \mathrm{O}_{2}$ (Fig 3a and Supplementary Fig 4a). Next, we performed functional experiments to further validate knockout strategy. Lactate secretion was only abolished on double LDHA/B KO cells (Fig 3b). LDH activity was decreased in LDHA, slightly decreased in LDHB single KO cells, but massively decreased in double KO cells (Supplementary Fig 4b). LDHB activity was not detected in LDHB or LDHA/B KO cells (Supplementary Fig 4c). Next, we transduced the lactate-sensitive FRET biosensor into all P3 cells to detect intracellular lactate levels (Supplementary Fig 4d) ${ }^{32}$. Basal lactate concentrations were higher in control, LDHA or LDHB KO cells than in double LDHA/B KO cells (Fig 3c). Adding oxamate, a potent LDH inhibitor that also induces lactate efflux by MCT trans-acceleration ${ }^{33}$, followed by a MCT blocker cocktail (diclofenac and AR-C155858) unveil that basal lactate concentrations are higher in control, LDHA or LDHB KO cells compared to double LDHA/B KO cells, are result explained by decreased lactate production (Fig 3c). Spheroid growth was next monitored over one week at $1 \% \mathrm{O}_{2}$ and showed that double LDHA/B KO strongly reduced spheroid growth, unlike LDHA KO that moderately decreased spheroid growth and LDHB KO that had no effect (Fig 3d, left panel). This was associated with a strong ethidium homodimer-1 staining for visualization of apoptotic events (Fig 3d, right panel). This cell death signature was also supported by a high Annexin-V staining in double KO cells detected by cytometry (Supplementary Fig 4e). 
The invasion capacity was analyzed in all cell lines and a moderate decrease in invasion was observed in LDHA KO in both P3 (Fig 3e) and BL13 cells (Supplementary Fig 4f). Nevertheless, LDHB KO P3 but not LDHB KO BL13 cells had higher invasive capacities (Fig. 3e and Supplementary Fig 4f). For LDHA/B KO cells, under $0.1 \% \mathrm{O}_{2}$, the invasive capacity dropped by $75 \%$ for P3 cells (Fig 3e), and by $50 \%$ for BL13 cells (Supplementary Fig 4f).

Spheroids from single and double LDHA/B KO cells were injected into immunodeficient mice to evaluate in vivo tumor development and survival (Fig 3f). No morphological differences were observed between control and single LDH KO (LDHA KO or LDHB KO) tumors (Fig 3g). However, double LDHA/B KO tumors were much smaller and less invasive than control tumors (Fig $3 \mathbf{h}$ ), which correlated with an increase in mouse survival, in both, the P3 (Fig 3i) and the BL13 model (Supplementary Fig 4i). Only a small increase in mouse survival was observed in the LDHA KO group, and, surprisingly, a drastic decrease in survival in LDHB KO group (Fig 3i), the latter was most likely due to haemorrhages at the tumor site. Vascular endothelial growth factor (VEGF), the main inducer of neoangiogenesis by tumor cells, was quantified by ELISA and an increase was only observed in LDHB KO cells (Supplementary Fig 4g). The VEGF inhibitor bevacizumab (bev) was then injected in mice bearing single or double LDHA/LDHB KO P3 tumors. An increase in survival was observed in mice harbouring bev-treated LDKB KO tumors and treated with bev, similar to control animals (Supplementary Fig 4h). Increased survival was also seen in mice with double LDHA/B KO tumors, while no difference was observed in mice with control or LDHA KO tumors

(Supplementary Fig 4h).

\section{Metabolic switch in double LDHA/B KO cells under hypoxia delineates vulnerabilities}

To trace metabolic fluxes at $0.1 \% \mathrm{O}_{2}$, cells were starved and infused with $\left[{ }^{13} \mathrm{C}_{6}\right]$ glucose for 24 or 48 hours. Principal component analysis (PCA), defining global RNA regulation, showed that metabolism of double LDHA/B KO cells switched after 48 hours at $0.1 \% \mathrm{O}_{2}$ when compared to control or single KO tumors (Fig 4a). Exometabolome of double LDHA/B KO cells strongly differed from other cells, by a decrease in glucose consumption and an absence of lactate production (Fig 4b). In addition, pyruvate and its derivates (acetate and formate) were only secreted in double LDHA/B KO cells (Fig 4b).

RNA sequencing was then performed on the same cells under 21 or $0.1 \% \mathrm{O}_{2}$, and confronted with metabolomics data. Metabolograms were used to integrate transcriptomics and metabolomics data as previously described ${ }^{34}$. The changes of each metabolite/transcript pair were determined and average metabolome/transcriptome variations of a metabolic pathways were visualized by comparing two conditions (Fig 4c). Based on global results showed previously (Fig 4a), metabolograms were built by analyzing total metabolite abundances in P3 control cells at $21 \%$ and while adapting at $0.1 \% \mathrm{O}_{2}$ (Fig 4c, left panels, and supplementary Fig 5), and compared to P3 control and P3 double LDHA/B KO cells at 21\% (Fig 4c, middle panels, and supplementary Fig 5) or at $0.1 \% \mathrm{O}_{2}$ (Fig 4c, right panels, and supplementary Fig 5). When changes in consensus gene expression were analyzed, differences in 
glycolysis or oxidative phosphorylation for all comparisons were seen, but only negligible variations for amino acid synthesis (Fig 4c). Significant variations in the majority of pathways from the metabolome perspective were observed for most of the comparisons (Fig 4c). For P3 control cells, the majority of transcripts were related to glycolysis and showed increases in expression at $0.1 \% \mathrm{O}_{2}$ when compared to $21 \% \mathrm{O}_{2}$ (18 up, 12 down), whereas the majority of metabolites significantly decreased at $0.1 \% \mathrm{O}_{2}(0$ up, 4 down) (Fig 4c, left panels). Metabolograms were heterogeneous when comparing changes between P3 control and double LDHA/B KO cells at (1) $21 \%$ and (2) at $0.1 \% \mathrm{O}_{2}$ (Fig 4c, middle and right panels). P3 double LDHA/B KO cells had a strongly modified metabolism, as seen by the increase in all metabolites and transcripts related to glycolysis and oxidative phosphorylation, especially under $0.1 \% \mathrm{O}_{2}$ (Fig $4 \mathrm{c}$ ).

Since metabolograms are based on the total metabolite abundances, metabolite abundance was calculated after $\left[{ }^{13} \mathrm{C}_{6}\right]$ glucose labelling using the fractional contribution and was incorporated into the central pathway of the carbon metabolic network map obtained from previous comparisons (Fig 4c). To explore the relationship between transcriptomic and metabolomic data, transcript levels of metabolismrelated genes were incorporated into the central carbon metabolic network pathway map (Fig 4d and supplementary Fig 6a-b). At 0 h, the difference between P3 double LDHA/B KO versus control cells was low to non-significant (Supplementary Fig 6 b). The detailed metabolic map depicts the increase in abundance for the majority of metabolites after $\left[{ }^{13} \mathrm{C}_{6}\right]$ glucose labelling in double KO LDHA/B cells versus control cells for the glycolytic and the oxidative phosphorylation pathways (Fig 4d). Fourteen transcripts from the glycolytic pathway (HK1, HK2, GPI, PFKB3, PFKB4, ALDOC, TPI1, PGK1, ENO1, ENO2 and PKM), and from the Krebs cycle (ACO2, SUCLG1and SDHB) were upregulated, while 6 were downregulated (GAPDHS, LDHA, LDHB, DLAT, SDHD, CS) (Fig 4d). These findings indicate that, under hypoxia, double LDHA/B KO deregulates cell metabolism at many levels. Global transcripts at $0.1 \% \mathrm{O}_{2}$ were increased in $\mathrm{P} 3$ control cells when compared to $21 \% \mathrm{O}_{2}$ (Supplementary Fig $6 \mathrm{c}$, upper panels), impacting metabolism, and in particular glycolysis (HK2, ENO1, ENO2, GAPDH, PKM, LDHA). At $21 \% \mathrm{O}_{2}$, major biological pathways are related to membrane and organelle dynamics during cell division and cell cycle regulation, at the contrary, in more unfavorable condition at $0.1 \% \mathrm{O}_{2}$, pathways are involved to low oxygen adaptation and maintenance of energy production (Supplementary Fig $\mathbf{6 c}$, upper panels). Hypoxic stress has been found to decrease the nucleotide pool ${ }^{35}$. Indeed, the abundance of nucleotides was lower while fractional contribution was enriched suggesting a continuous but limited turnover (Supplementary Fig 7). Under $0.1 \% \mathrm{O}_{2}$, the transcription profiles were more enriched in double LDHA/B KO cells when compared to P3 sgControl cells (Supplementary Fig 6c, lower panels). This was also seen at $21 \%$ $\mathrm{O}_{2}$ (Supplementary Fig $6 \mathbf{c}$, middle panels). The differences in abundance and isotopolog contribution between control and double KO LDHA/B cells were less pronounced in $21 \% \mathrm{O}_{2}$. In hypoxia, the abundance in nucleotide monophosphates (AMP, GMP and UMP) was also higher in double KO LDHA/B cells (Supplementary Fig 7). The fractional contribution of purines and pyrimidines mainly consisted in $\mathrm{m}+5$ labeling in double KO LDHA/B cells whereas $m+5, m+7$ and $m+8$ labeling was seen in control cells. 
Double LDHA/B KO cells reorganize their mitochondrial respiratory chain to support growth under $0.1 \%$ $\mathrm{O}_{2}$ concentration

To corroborate the RNA sequencing results (Fig 4c-d and supplementary Fig 6a), we exposed P3 cells to 21 or $0.1 \% \mathrm{O}_{2}$ and analyzed the subunits of the mitochondrial respiratory chain by Western blot. NDUFB8, SDHA, UQCRC2, COXII protein levels increased upon $0.1 \% \mathrm{O}_{2}$ in double LDHA/B KO cells, but only NDUFB8 and COXII at $21 \% \mathrm{O}_{2}$ (Fig 5a). Expression of the subunit V ATP5A complex did not change in high or low oxygen conditions (Fig 5a). For single LDHB or LDHA KO cells, only minor changes were observed (Fig 5a). Immunostaining of mitochondria networks revealed an increase in the mitochondrial mass and modifications in network shape, defined as aspect ratio, only in double LDHA/B KO cells (Fig 5b). Under uncoupling conditions, double LDHA/B KO cells possess higher respiratory capacity than the other cells (Fig 5c). Two in vivo strategies were employed to inhibit these metabolic adaptations. The double LDHA/B KO tumors were challenged either with the respiratory complex I inhibitor phenformin or by irradiation (Fig 5d). Phenformin injections did not improve survival of KO LDHA/B tumor bearing mice when compared to control tumors (Fig 5e), while irradiation massively increased survival in mice with double LDHA/B KO tumors (Fig 5e).

\section{Use of anti-epileptic drug targeting LDH activity efficiently reduces GBM development}

Mice were next treated with the anti-epileptic drug stiripentol, which inhibit LDH activity and is capable to cross the blood-brain barrier ${ }^{36}$. Importantly, this drug has already been used in preclinical therapy studies in vivo in mice ${ }^{26}$ (Fig 6a). We first validated LDH inhibition in vitro in P3 GBM cells by intracellular lactate recording with the FRET biosensor Laconic, finding that lactate level decreased and its production was inhibited a $50 \%$ after $24 \mathrm{hrs}$ treatment with $500 \mu \mathrm{M}$ of stiripentol (Fig $6 \mathrm{~b}$ ). This was correlated with a drastic decrease of basal and uncoupled respiration in pre-treated P3 cells (Fig 6c). Furthermore, stiripentol significantly reduced P3 spheroid proliferation (Fig 6d) and invasion at $0.1 \% \mathrm{O}_{2}$ (Fig 6e). To test stiripentol anti-cancer properties in vivo, intraperitoneally injection of either $100 \mathrm{mg} / \mathrm{kg}$ of stiripentol (stiri) or $10 \mathrm{mg} / \mathrm{kg}$ of bevacizumab (bev) alone, or in combination (combo) in animals implanted with P3 cells were then carried-out (Fig 6f). Stiripentol treatment had some effect on mouse survival when administered alone but its combination with bevacizumab treatment significantly increased survival (Fig 6f). Immunohistochemistry analysis showed reduction in tumor size and invasion when treated with the combinatory regimen (Fig $\mathbf{6 g}$ ).

\section{Discussion}

A common property of many solid cancers including GBMs, is upregulation of glycolysis ${ }^{1-3}$ and it has been postulated that blocking glycolysis could be a useful target in fighting of this aggressive disease. Our study pinpoints lactate as one of the main metabolic drivers in glioblastoma, through both lactate dehydrogenases (LDHA and LDHB) activities to support tumor development and invasion. 
Histological analysis of LDHA and LDHB expression demonstrated a clear pattern with LDHA predominantly expressed in the central hypoxic area, confirming our previous data ${ }^{37}$, whereas LDHB was expressed in peripheral and invasive tumor areas. Only a few cells were found positive for LDHA in peripheral and invasive areas in the corpus callosum. This pattern was also confirmed in the collagenembedded invasive spheroid model where LDHA was mainly found in the hypoxic core of spheroid ${ }^{28}$ and in a few invasive cells. LDHB is exclusively localized at spheroid borders and in invasive cells. This observed differential spatial distribution was reinforced by analyzing a single-cell database which include RNAseq data from central and invasive GBM regions ${ }^{29}$. These results demonstrate that LDHA or LDHB spatial distribution echoes the expression of the lactate transporters MCT1 and MCT $4^{31}$, supporting the hypothesis of lactate shuttle between cells, as described in the astrocyte-neuron lactate shuttle (ANLS).

We evidenced that lactate, as a single carbon source being retro-converted into pyruvate, is able to fuel the TCA cycle via upregulation of oxidative phosphorylation, an observation that become evident when inhibiting the respiratory chain complex I inhibition with rotenone. Our data also show that lactate alone can regulate tumor invasion in cells starved for most carbon sources. When glucose is present, lactate and acidification (by $\mathrm{HCl}$ ) regulate $\mathrm{GBM}$ invasion ${ }^{22}$. Acidification of the microenvironment participates to cell invasion by upregulating many factors such as TGF $\beta^{22}$. Labelling of starved GBM stem-like cells with $\left[{ }^{13} \mathrm{C}_{3}\right]$ lactate quickly generated pyruvate which fuels the TCA cycle, or converted into alanine which is secreted into the extracellular space. The TCA cycle plays a central role in energy metabolism through acetyl-CoA oxidation but has also functions in biosynthetic pathways (e.g. non-essential amino acids or fatty acids $)^{38}$. As the TCA cycle cannot fully oxidize all intermediates, known as cataplerosis, these were removed from the cycle and secreted by the cells mainly in the form of glutamine and asparagine. Moreover, exogenous lactate provides an important and rapid carbon pool for citrate-mediated fatty acid biosynthesis ${ }^{39,40}$. Consistent with a previous study ${ }^{39}$, we observed a smaller $m+2$ isotopolog contribution of a-ketogluratate and succinate compared to citrate. Oddly enough, $m+2$ isotopolog contribution of glutamate and malate tend to be similar to citrate.

Single KO of LDHA does not have a strong effect in the stem-like GBM cell models used in this study, which is different to LDHA KO neuroblastoma cells described in another publication ${ }^{41}$. When LDHA is absent, compensatory mechanisms are observed in our study, even under low oxygen conditions. Surprisingly, KO LDHB cells were more aggressive on in vitro and in vivo experiments, an observation that may be partially explained by an accumulation of extracellular lactate and higher production of VEGF, inducing a strong vascular response and lower survival of implanted animals. LDHB knockout was previously characterized to be linked to autophagy, and reduction of cell proliferation ${ }^{24}$, which was not observed in our study. Double LDHA/B KO induced a strong effect on proliferation and invasion and was also linked to an increase in apoptosis, thus improving mouse survival via reduction of the tumor mass. While complementary roles of LDHA and LDHB in brain tumor development have been reported in a neuroblastoma model ${ }^{41}$, double KO in GBM stem-like cells demonstrated a strong effect on tumor development via lactate production and consumption. In an elegant publication, Zdralevic and colleagues 
showed that LDHA/B ablation impairs melanoma and colon adenocarcinoma growth ${ }^{42}$, but with less difference for melanoma between single LDHA KO and LDHA/B KO when compared to our study. Cerebral tumors rely on low oxygen concentrations, which can partially explain these differences between the models. As lactate has been shown to be immunosuppressive ${ }^{43}$, we are, at epresent, investigating macrophages/microglia activation in our in vivo models, as they represent the main immune cells in glioblastoma ${ }^{44}$. GBM stem-like cells evolve in a different microenvironment than melanoma cells or adenocarcinoma cells and, thus, metabolic regulations during tumor growth may be different. Furthermore, the experiments were not done under the same oxygen concentrations than in our study.

Accumulation and excretion of incompletely catabolized intermediates could reflect hyperactive metabolism ${ }^{45}$ or a blockade of essential pathways. The best example is the Warburg effect, where a high glycolytic rate lead to pyruvate excess that cannot be consumed by mitochondria, resulting in significant lactate production and secretion. In addition, GBM LDHA/B KO cells lost the ability to maintain the redox balance under anaerobic conditions. Therefore, the double knock-out globally impacts the cellular metabolism and this is associated with a decrease of glucose consumption, an accumulation of the majority of intermediates from glycolysis and TCA and excretion of formate or acetate. One possible explanation for fermentative by-product overflow is that the pyruvate-derived acetate is produced to maintain a lower redox potential to compensate $\mathrm{LDH} \mathrm{KO}{ }^{46}$. We also observed a strong effect on the expression of the respiratory complex in LDHA/B KO cells in hypoxia to counteract the metabolic stress in these extreme conditions. To increase apoptosis in LDHA/B KO cells, an inhibitor of respiratory complex I was used. However, this treatment failed to increase apoptosis which was possibly due to the compensation by other respiratory complexes or alternative metabolic pathways such fatty acid oxidation. After cranial irradiation, on the contrary, a strong increase in mouse survival was observed.

In a recent study, the use of anti-epileptics as anticancer drugs has been proposed, and this could be beneficial for the treatment of GBM patients ${ }^{47}$. We therefore took advantage of the use of the stiripentol, an antiepileptic drug approved for the treatment of Dravet Syndrome because is also potently inhibits both LDHA and LDHB activity ${ }^{26}$. Our results demonstrate that stiripentol coupled with an antiangiogenic drug induced a significant therapeutic response with inhibition of tumor growth and tumor cell invasion.

All in all, we conducted herein a detailed analysis of the metabolic consequences of the deletion or inhibition of LDH enzymes in GBM cells and demonstrate that targeting of both LDHA and LDHB is required for efficient inhibition of tumor growth. These results are of great translational significance because these enzymes may represent interesting candidates for further therapeutic development in GBM.

\section{Declarations}

\section{Author contributions:}


Acknowledgements

\section{Potential financial conflict of interest}

The authors state no financial conflict of interest.

\section{Materials And Methods}

\section{Ethical issues}

Male RAGy2C ${ }^{-/-}$mice were housed and treated in the animal facility of Bordeaux University. All animal procedures have been done according to the institutional guidelines and approved by the local ethics committee (agreement number: A5522). The collection of biopsy tissue was approved by the regional ethical committee at Haukeland University Hospital, Bergen, Norway (REK 013.09), and by Humanitas Hospital (Milan, Italy).

\section{Antibodies and reagents}

The detailed information of primary and secondary antibodies used in this study is listed in Reagent List. For immunofluorescence, DAPI was used to stain DNA in blue, Phalloidin Rhodamine was used to stain cytoskeleton in red. Calcein-AM and ethidium homodimer-1 was directly added to cultures in their usual media. Calcein-AM is converted into green fluorescent calcein by intracellular esterase and is an indicator of cell viability. Injured or dead cells take up ethidium homodimer-1 which stained the DNA in red. Spheroids are incubated in these reagents for $45 \mathrm{~min}$ at $37^{\circ} \mathrm{C}$ at which time images were directly photographed. Others reagents and products are listed in Reagent List.

\section{Cell culture}

Patient-derived GBM P3 and BL13 cells were cultured in NeuroBasal medium (NBM) supplemented with B27, heparin $(100 \mathrm{U} / \mu \mathrm{L}), 20 \mathrm{ng} / \mathrm{mL}$ basic FGF and Penicillin - Streptomycin $(1000 \mathrm{U} / \mathrm{mL})$ at $37^{\circ} \mathrm{C}$ in $5 \%$ $\mathrm{CO}_{2}$ incubator. Stable cell lines were generated by infecting lentiviral particles. For generation of lentiviral particles, HEK293T cells were transfected with the lentiCRISPR v2 vector with sgRNA, psPAX2 (packaging construct) and pMD2.G (viral envelope). The culture medium was replaced by Opti-MEM with $20 \mathrm{mM}$ HEPES 6 hours after transfection. After 2-3 days of culture, the supernatant was collected, passed through a $0.22 \mu \mathrm{m}$ syringe filter. The virus-containing media supplemented with $8 \mu \mathrm{g} / \mathrm{mL}$ polybrene was used to infect P3 and BL13 cells. The infected cells were selected in media with puromycin $(0.75 \mu \mathrm{g} / \mathrm{mL})$ or blasticidin $(10 \mu \mathrm{g} / \mathrm{mL})$ and the LDHA or/and LDHB deletion was assessed by Western blot. 


\section{sgRNA constructs}

LDHA and LDHB sgRNAs were designed online (http://crispr.mit.edu/). The target sequences were 5'TGCGAATACGCCCACGCGATGGG-3' for Control, 5'-CCGATTCCGTTACCTAATGGGGG-3' for LDHA, and 5'AAGATCACTGTAGTGGGTGT-3' for LDHB. The sgRNAs were then cloned into the lentiCRISPR v2 vector by a BsmBI digestion.

\section{Spheroid experimental assays}

Protocols are described into details in a previous article ${ }^{28}$. Briefly, P3 and BL13 spheroids of uniform size were prepared from $10^{4}$ dissociated cells in NBM with $0.4 \%$ methylcellulose in a 96 -well round-bottom plate. The spheroids were used after 3 days of formation. For spheroid growth, each spheroid was washed with PBS and individually replaced in fresh complete neurobasal medium with $0.4 \%$ methylcellulose supplemented with different treatments in a 96-well round bottom plate. For spheroid invasion, spheroids were washed in PBS and individually included in a 96-well flat-bottom plate in $100 \mu \mathrm{L}$ of type I collagen matrix prepared on ice with $1 \mathrm{mg} / \mathrm{mL}$ collagen type I and $\mathrm{NaOH}$ (initial concentration: $1 \mathrm{M}$, volume added correspond to 0.023 volume of collagen type I). The matrix was incubated at $37^{\circ} \mathrm{C}$ for 30 minutes and then, complete neurobasal medium with different treatments were added over the matrix.

\section{Intracranial implantation}

For in vivo experiments, 5 spheroids of P3 or BL13 knock-out for LDHA, LDHB or LDHA/B (only LDHA/B for BL13) and control cell lines were stereotactically implanted into the brains of immunodeficient RAGy2C ${ }^{-/-}$mice ( $n=8$ for survival or $n=5$ for stop at a given point, 7-9-week-old) housed and treated in the animal facility of Bordeaux University ("Animalerie Mutualisée Bordeaux"). The animals were anesthetized with ketamine $(1.5 \mathrm{mg} / \mathrm{Kg})$ and xylazine $(150 \mu \mathrm{g} / \mathrm{Kg})$ and a burrhole was drilled $2.2 \mathrm{~mm}$ to the left of the bregma, and the spheroids were implanted into the cerebral cortex at $3 \mathrm{~mm}$ depth using a $10 \mu \mathrm{L}$ Hamilton syringe. An analgesic procedure was applied with the subcutaneous injection of buprenorphine $(0.1 \mathrm{mg} / \mathrm{kg}$, once $10 \mathrm{~min}$ before and once 12 to $24 \mathrm{~h}$ after implantation). After 4 days, started the bevacizumab injections (10 mg/kg ip), and/or stiripentol (100 mg/kg ip), or phenformin (50 $\mathrm{mg} / \mathrm{kg}$ ip) injection 3 times a week. The animals were euthanized by cervical dislocation at the end of the experiment and brains removed for further histological and immunohistological analyses.

\section{Histological samples preparation}


For frozen process, mice brains were placed in a cryotube after extraction, directly frozen in liquid nitrogen without fixation and stored at $-80^{\circ} \mathrm{C}$. Sections $(10 \mu \mathrm{m})$ were prepared using a cryostat (CM1900, Microsystems) and mounted on slides. Before immunolabeling process (see below), cryo-sections were dried at room temperature for 10 minutes. They were fixed with $4 \%$ paraformaldehyde (PFA) for 15 min at room temperature, then washed 3-times in phosphate-buffered saline (PBS). For paraffin process, mice brains or other samples were fixed with 4\% PFA for $15 \mathrm{~min}$ at room temperature, then washed 3-times in PBS. Samples were dehydrated serially in 70,96 , and $100 \%$ ethanol then toluene, and paraffinized. Sections $(10 \mu \mathrm{m})$ were prepared using a microtome (Leica) and mounted on slides. Before immunolabeling process (see below), paraffin-embedded sections were deparaffinized in toluene and hydrated serially in 100,96 , and $70 \%$ ethanol and distilled water then for $1 \mathrm{~h}$ at $95^{\circ} \mathrm{C}$ in citrate buffer before staining procedure.

\section{Immunolabeling process}

Samples were permeabilized with $0.1 \%$ Triton X-100 in PBS for 15 min, quickly washed in PBS, blocked with $1 \%$ bovine serum albumin and $2 \%$ fetal donkey serum (FDS) in PBS (blocking buffer) for $1 \mathrm{~h}$. Samples were incubated with primary antibodies in blocking buffer overnight at $4^{\circ} \mathrm{C}$, followed by 3-time PBS wash and incubation with secondary antibodies in blocking buffer for $1 \mathrm{~h}$, for staining DNA or/and cytoskeleton, samples were treated with DAPI or/and Phalloidin Rhodamine, respectively. Samples were washed 3-times in PBS and were mounted using Prolong Gold antifade reagent.

\section{Western blot}

Cells were washed twice in PBS and, depending on the analysis, two procedures were used for protein lysis. (1) Cells were lysed in RIPA buffer $(10 \mathrm{mM}$ Tris-HCl, pH 7.4, $150 \mathrm{mM} \mathrm{NaCl}, 0.5 \%$ NP-40, $1 \%$ TritonX100, 1 mM EDTA) containing proteases and phosphatases inhibitors. Protein concentrations were determined using bicinconinic acid assay. Cell lysates were resuspended in Laemmli Buffer (LB - 62.5 $\mathrm{mM}$ Tris, $10 \%$ glycerol, $2.5 \%$ SDS, $5 \% \beta$-mercaptoethanol, $\mathrm{pH}$ 6.8). (2) Cells were directly lysed in LB. All protein extracts ( 1 and 2 ) were separated on an acrylamide gel (10\% for LDHA and B, 4-12\% precasted gels (Invitrogen) for OxPhos proteins) and transferred to a nitrocellulose membrane. After blocking in blocking buffer for 1 hour at room temperature, membranes were incubated with different primary antibodies overnight at $4^{\circ} \mathrm{C}$. After 3 washes in TBST (20 mM Tris, $0.5 \mathrm{~N} \mathrm{NaCl}, 0.1 \%$ Tween-20, $\mathrm{pH}=8$ ) and 1 in TBS (20 mM Tris, $0.5 \mathrm{~N} \mathrm{NaCl}, \mathrm{pH}=8)$, membranes were incubated with IR-Dye 680 or 800 labelled secondary antibodies, or secondary antibodies coupled with HRP.

\section{Image acquisition and analysis}


For Western Blot analysis, membranes were imaged using Odyssey infra-red scanner (LI-COR). The densitometry of proteins was quantified using Image Studio Lite Software with normalization against tubulin or vinculin. Uncropped immunoblot are depicted in Supplementary Figure 8. For cell or spheroid experiment assays, images were acquired with an Eclipse Ti Nikon microscope equipped with NIS (Nikon imaging element) software based on $\mathrm{x} 4, \mathrm{x} 10$ or $\mathrm{x} 20$ objective lens (Nikon), a Hamamatsu Digital CCD C10600-10B camera. Images were acquired in brightfield or/and color filters (red/green) at the plane level where the contrast is the most pronounced. Fiji software with different open-access macros was used for image analysis (GitHub). Images of the spheroid growth were acquired at $0 \mathrm{~h}$ and every $24 \mathrm{~h}$ (total duration dependent on the experiments), growth was quantified as the percent of change of the spheroid area relative to $0 \mathrm{~h}$. Images of the spheroid invasion were acquired at $24 \mathrm{~h}$, invasion was quantified as the invasive area (total area - core area) normalized to the core area, and expressed as a fold change relative to the control group for each independent experiment. For samples from immunolabeling process, images were acquired with confocal microscope (Nikon Elipse Ti) or epifluorescence microscope (Nikon). The pipeline analysis of mitochondrial morphology and mass was adapted in Fiji software from Koopman et $\mathrm{al}^{48}$. The slide scanner was a Nanozoomer 2.0HT with fluorescence imaging module (Hamamatsu Photonics France) using objective UPS APO 20X NA 0.75 combined to an additional lens $1.75 \mathrm{X}$, leading to a final magnification of 35X. Virtual slides were acquired with a TDI-3CCD camera. Fluorescent acquisitions are done with a mercury lamp (LX2000 200W - Hamamatsu Photonics, Massy, France) and the set of filters adapted for DAPI, and/or GFP/Alexa 488, and/or Alexa 568 and or Alexa 647/Cy5 fluorescence.

\section{Sample preparation and transcriptomic profiling}

Transcriptomic sequencing was performed in collaboration with Core Unit for Molecular Tumor Diagnostics (CMTD), National Center for Tumor Diseases (NCT), Dresden-Germany. Details are included in the Supplemental Experimental Procedures. The R package DESeq $2^{49}$ was used to identify differentially expressed genes using a stringent threshold: absolute value of Log2 Fold Change (LFC) $>1$ and $p$-value adjusted for multiple testing ( $\mathrm{p}$-adjust) $<0.01$. KEGG pathways enrichment analysis was done with the R package ClusterProfiler ${ }^{50}$. Gene Ontology (GO) terms enrichment analysis was done with the $\mathrm{R}$ package BACA with the EASE score set at 0.01. Only GO terms from Biological Pathways level 5 were used for this analysis. GO terms were clustered to get annotation clusters with a similarity of genes greater than 0.85 . Finally, read counts were transformed using variance stabilizing transformations (VST).

\section{Sample preparation and metabolic profiling}


Metabolomic profiling was performer in collaboration with MetaboHUB-MetaToul $\left(\left[{ }^{13} \mathrm{C}\right]_{6}\right.$ Glucose) and VIB Metabolomics $\left(\left[^{13} \mathrm{C}\right]_{6}\right.$ Lactate). Details are included in the Supplemental Experimental Procedures. The Principal Component Analysis (PCA) was done on the total geometric mean abundance of each metabolite in each condition. PCA is realized with the dudi .pca function from the R package « ade4 » and visualized with ggplot. Metabolite abundance between two conditions was analyzed using the nonparametric Mann-Whitney test with a Benjamini-Hochberg correction for multiple comparisons. For visualisation purposes, comparisons between two conditions may be Log2-transformed.

\section{Metabolomic and transcriptomic representation}

Metabolome and transcriptome were independently analyzed (see above). Due to the difficulty in examining detailed metabolic networks using several cell lines and conditions, two representations are proposed. The first one, called metabologram ${ }^{49}$, have the ability to visualize data at pathway level relative to the second one, which is the classical detailed network map of central carbon. In order to avoid redundancy of data, the metabologram showed all the isotope abundances in metabolites and the network map showed only the $\left[{ }^{13} \mathrm{C}\right]$-labelled abundance in metabolite in order to trace the pathway of $\left[{ }^{13} \mathrm{C}\right]$-initial metabolite. For each pathway and after the stringent threshold based on log2 FC and p-value $(p<0.05)$, transcripts are selected according to the KEGG pathway database.

Intracellular lactate recording. Cultured P3 spheroids were gently dissociated and seeded on $18 \mathrm{~mm}$ glass coverslips treated with Matrigel. Constructs coding for the lactate-sensitive FRET biosensor Laconic ${ }^{32}$ have been described previously and are available through Addgene (Plasmid \#44238). Adenoviral vectors encoding the FRET biosensor was custom made by Vector Biolabs. The lactate-sensitive biosensor Laconic was expressed by exposing cells to $1 \times 10^{6} \mathrm{PFU}$ of adenoviral particles (serotype 5) overnight. After 48-72 hrs post infection, cells were imaged on wide-field mode with an inverted LEICA DMI6000B microscope (Leica Microsystems, Germany) equipped with a motorized stage Scan IM (Märzhäuser, Germany), a 40X oil-immersion objective (NA 1.25), a excitation system Lumencor spectra 7 (Lumencor, US) and a Coolsnap HQ2 CDD camera (Photometrics, US). Cells were superfused with an imaging solution consisting of (in mM): 10 HEPES, $112 \mathrm{NaCl}, 24 \mathrm{NaHCO}, 3 \mathrm{KCl}, 1.25 \mathrm{MgCl} 2,1.25 \mathrm{CaCl} 2,10$ glucose and bubbled with air $/ 5 \% \mathrm{CO}_{2}$ at $37{ }^{\circ} \mathrm{C}$ with a constant flow of $3 \mathrm{ml} / \mathrm{min}$. For fluorescent ratio measurements, cells expressing Laconic were excited at $430 \mathrm{~nm}$ for $0.05-0.1 \mathrm{~s}$ and emission collected with band pass filters mounted on a motorized filter wheel, at $465-485 \mathrm{~nm}$ for mTFP and 542-556 nm for Venus, with image acquisition every $10 \mathrm{~s}$. The ratio between mTFP and Venus was computed and is proportional to the intracellular lactate level. To quantify differences in lactate level, biosensor occupancy was computed as a proxy of intracellular lactate level with the following equation: Occupancy = (R$\mathrm{Rmin}) /(\mathrm{Rmax}-\mathrm{Rmin})$, in which R: basal mTFP/Venus ratio (before any drug treatment), Rmin: steady state mTFP/Venus ratio induced by oxamate $(6 \mathrm{mM})$, Rmax: steady state mTFP/Venus ratio induced by MCTs 
block ( $1 \mu \mathrm{M}$ AR-C155858 and $1 \mathrm{mM}$ diclofenac). Lactate production rate was computed by fitting a linear rate to the first minutes of lactate accumulation during MCTs block. Fluorescence ratios were normalized to Rmin before quantifications.

\section{Statistical analysis}

No statistical methods were used to predetermine sample size. The experiments were not randomized and investigators were not blinded to allocation during experiments and outcome assessment. GraphPad Prism software and RStudio was used for analyses. Equality of variances was made with a F-test for two groups and Brown-Forsythe test for at least three groups. According to the homoscedasticity, comparisons of two groups were made by an unpaired two-tailed $t$-test or a Mann-Whitney test. Comparisons of at least three groups were performed using a one-way ANOVA followed by a Dunnett's or Tukey's multiple comparisons test or Kruskal-Wallis test followed by a Dunn's multiple comparisons test. A two-way ANOVA test was used to compare two or more than two groups with two variables or more than two variables (e.g. $21 \%$ and $0.1 \% \mathrm{O}_{2}$ ) or repeated measures at different times followed by a Tukey's multiple comparisons test. Survival analyses were made using log-rank Mantel-Cox test. All the tests used were indicated in the figure legends. $P$ values $<0.05$ were considered statistically significant.

\section{Data availability}

All data are available within the Article and Supplementary Files, or available from the corresponding authors on reasonable request. Transcriptomics data are available from the European Nucleotide Archive: PRJEB45718.

\section{References}

1. Giese, A., Bjerkvig, R., Berens, M. E. \& Westphal, M. Cost of Migration: Invasion of Malignant Gliomas and Implications for Treatment. J. Clin. Oncol. 21, 1624-1636 (2003).

2. Neftel, C. et al. An Integrative Model of Cellular States, Plasticity, and Genetics for Glioblastoma. Cell 178, 835-849.e21 (2019).

3. Vollmann-Zwerenz, A., Leidgens, V., Feliciello, G., Klein, C. A. \& Hau, P. Tumor Cell Invasion in Glioblastoma. Int. J. Mol. Sci. 21, (2020).

4. Scherer, H. J. THE FORMS OF GROWTH IN GLIOMAS AND THEIR PRACTICAL SIGNIFICANCE. Brain $63,1-35$ (1940).

5. Daubon, T. et al. Deciphering the complex role of thrombospondin-1 in glioblastoma development. Nat. Commun. 10, 1146 (2019). 
6. Fack, F. et al. Bevacizumab treatment induces metabolic adaptation toward anaerobic metabolism in glioblastomas. Acta Neuropathol. (Berl.) 129, 115-131 (2015).

7. Venkataramani, V. et al. Glutamatergic synaptic input to glioma cells drives brain tumour progression. Nature 573, 532-538 (2019).

8. Gatenby, R. A. \& Gillies, R. J. Why do cancers have high aerobic glycolysis ? Nat. Rev. Cancer 4, 891899 (2004).

9. Warburg, O. On the Origin of Cancer Cells. Science 123, 309-314 (1956).

10. Liberti, M. V \& Locasale, J. W. The Warburg Effect: How Does it Bene fi t Cancer Cells ? Trends Biochem. Sci. 41, 211-218 (2016).

11. Altenberg, B. \& Greulich, K. O. Genes of glycolysis are ubiquitously overexpressed in 24 cancer classes. Genomics 84, 1014-1020 (2004).

12. Koukourakis, M. I. et al. Lactate dehydrogenase-5 (LDH-5) overexpression in non-small-cell lung cancer tissues is linked to tumour hypoxia, angiogenic factor production and poor prognosis. $\mathrm{Br}$. J. Cancer 89, 877-885 (2003).

13. Koukourakis, M. I., Giatromanolaki, A., Simopoulos, C. \& Sivridis, E. Lactate dehydrogenase 5 (LDH5) relates to up-regulated hypoxia inducible factor pathway and metastasis in colorectal cancer. $\mathbf{5}, \mathbf{2 5 -}$ 30 (2005).

14. Sheng, S. L. et al. Knockdown of lactate dehydrogenase A suppresses tumor growth and metastasis of human hepatocellular carcinoma. FEBS J. 279, 3898-3910 (2012).

15. Xie, H. et al. Targeting Lactate Dehydrogenase-A Inhibits Tumorigenesis and Tumor Progression in Mouse Models of Lung Cancer and Impacts Tumor-Initiating Cells. Cell Metab. 19, 795-809 (2014).

16. Fantin, V. R., St-Pierre, J. \& Leder, P. Attenuation of LDH-A expression uncovers a link between glycolysis, mitochondrial physiology, and tumor maintenance. Cancer Cel/ 9, 425-434 (2006).

17. Le, A. et al. Inhibition of lactate dehydrogenase A induces oxidative stress and inhibits tumor progression. Proc. Natl. Acad. Sci. 107, 2037-2042 (2010).

18. Goetze, K., Walenta, S., Ksiazkiewicz, M., Kunz-Schughart, L. A. \& Mueller-Klieser, W. Lactate enhances motility of tumor cells and inhibits monocyte migration and cytokine release. Int. J. Oncol. 39, 453463 (2011).

19. Rizwan, A. et al. Relationships between LDH-A, Lactate and Metastases in 4T1 Breast Tumors. Clin Cancer Res 19, 5158-69 (2013).

20. Baumann, F. et al. Lactate promotes glioma migration by TGF- b 2-dependent regulation of matrix metalloproteinase-2. Neuro-Oncol. 368-380 (2009) doi:10.1215/15228517-2008-106.

21. Colen, C. B. et al. Metabolic Targeting of Lactate Efflux by Malignant Glioma Inhibits Invasiveness and Induces Necrosis: An In Vivo Study 1. Neoplasia 13, 620-632 (2011).

22. Seliger, C. et al. Lactate-Modulated Induction of THBS-1 Activates Transforming Growth Factor (TGF) -beta2 and Migration of Glioma Cells In Vitro. PLOS One 8, e78935 (2013). 
23. Chesnelong, C. et al. Lactate dehydrogenase A silencing in IDH mutant gliomas. Neuro-Oncol. 16, 686-695 (2014).

24. Brisson, L. et al. Lactate Dehydrogenase B Controls Lysosome Activity and Autophagy in Cancer. Cancer Cell 30, 418-431 (2016).

25. Wang, J. et al. A reproducible brain tumour model established from human glioblastoma biopsies. 13, 1-13 (2009).

26. Sada, N., Lee, S., Katsu, T., Otsuki, T. \& Inoue, T. Targeting LDH enzymes with a stiripentol analog to treat epilepsy. Science 347, 1362-1367 (2015).

27. Daubon, T. et al. The invasive proteome of glioblastoma revealed by laser-capture microdissection. Neuro-Oncol. Adv. 1, vdz029 (2019).

28. Guyon, J. et al. A 3D Spheroid Model for Glioblastoma. J. Vis. Exp. JoVE(2020) doi:10.3791/60998.

29. Darmanis, S. et al. Single-Cell RNA-Seq Analysis of Infiltrating Neoplastic Cells at the Migrating Front of Human Glioblastoma. Cell Rep. 21, 1399-1410 (2017).

30. Sakadžić, S. et al. Two-photon high-resolution measurement of partial pressure of oxygen in cerebral vasculature and tissue. Nat. Methods 7, 755-759 (2010).

31. Allen, E. et al. Metabolic Symbiosis Enables Adaptive Resistance to Anti-angiogenic Therapy that Is Dependent on mTOR Signaling. Cell Rep. 15, 1144-1160 (2016).

32. San Martín, A. et al. A genetically encoded FRET lactate sensor and its use to detect the Warburg effect in single cancer cells. PloS One 8, e57712 (2013).

33. Contreras-Baeza, Y. et al. Monocarboxylate transporter 4 (MCT4) is a high affinity transporter capable of exporting lactate in high-lactate microenvironments. J. Biol. Chem. 294, 20135-20147 (2019).

34. Hakimi, A. A. et al. An Integrated Metabolic Atlas of Clear Cell Renal Cell Carcinoma. Cancer Cell 29, 104-116 (2016).

35. Hisanaga, K., Onodera, H. \& Kogure, K. Changes in levels of purine and pyrimidine nucleotides during acute hypoxia and recovery in neonatal rat brain. J. Neurochem. 47, 1344-1350 (1986).

36. Sada, N., Lee, S., Katsu, T., Otsuki, T. \& Inoue, T. Targeting LDH enzymes with a stiripentol analog to treat epilepsy. Science 347, 1362-1367 (2015).

37. Talasila, K. M. et al. The angiogenic switch leads to a metabolic shift in human glioblastoma. NeuroOncol. 19, 383-393 (2017).

38. Owen, O. E., Kalhan, S. C. \& Hanson, R. W. The Key Role of Anaplerosis and Cataplerosis for Citric Acid Cycle Function. J. Biol. Chem. 277, 30409-30412 (2002).

39. Chen, Y.-J. et al. Lactate metabolism is associated with mammalian mitochondria. Nat. Chem. Biol. 12, 937-943 (2016).

40. Minami, N. et al. Lactate Reprograms Energy and Lipid Metabolism in Glucose-Deprived Oxidative Glioma Stem Cells. Metabolites 11, 325 (2021).

41. Dorneburg, C. et al. LDHA in Neuroblastoma Is Associated with Poor Outcome and Its Depletion Decreases Neuroblastoma Growth Independent of Aerobic Glycolysis. Clin. Cancer Res. 24, 5772- 
5783 (2018).

42. Ždralević, M. et al. Double genetic disruption of lactate dehydrogenases $A$ and $B$ is required to ablate the 'Warburg effect' restricting tumor growth to oxidative metabolism. J. Biol. Chem. 293, 1594715961 (2018).

43. Fischer, K. et al. Inhibitory effect of tumor cell-derived lactic acid on human T cells. Blood 109, 3812-3819 (2007).

44. Müller, S. et al. Single-cell profiling of human gliomas reveals macrophage ontogeny as a basis for regional differences in macrophage activation in the tumor microenvironment. Genome Biol. 18, 234 (2017).

45. Meiser, J. et al. Increased formate overflow is a hallmark of oxidative cancer. Nat. Commun. 9, 1368 (2018).

46. Liu, X. et al. Acetate Production from Glucose and Coupling to Mitochondrial Metabolism in Mammals. Cell 175, 502-513.e13 (2018).

47. Jung, E. et al. Emerging intersections between neuroscience and glioma biology. Nat. Neurosci. 22, 1951-1960 (2019).

48. Koopman, W. J. H., Visch, H.-J., Smeitink, J. A. M. \& Willems, P. H. G. M. Simultaneous quantitative measurement and automated analysis of mitochondrial morphology, mass, potential, and motility in living human skin fibroblasts. Cytometry A 69A, 1-12 (2006).

49. Hakimi, A. A. et al. An Integrated Metabolic Atlas of Clear Cell Renal Cell Carcinoma. Cancer Cell 29, 104-116 (2016).

50. Yu, G., Wang, L.-G., Han, Y. \& He, Q.-Y. clusterProfiler: an R Package for Comparing Biological Themes Among Gene Clusters. OMICS J. Integr. Biol. 16, 284-287 (2012).

\section{Abbreviations}

3D, three dimensional; Bev, bevacizumab; Ivy GAP, Ivy Glioblastoma Atlas Project GBM, glioblastoma; GFP, green fluorescent protein; KO, knock-out; LA, lactic acid; LDH, lactate dehydrogenase; MCT, monocarboxylate transporter; OxPhos, oxidative phosphorylation; PDX, patient-derived xenografts; ROS, reactive oxygen species; sgRNA, single guide RNA; TCA, tricarboxylic acid; TCGA, Tissue Cancer Genome Atlas.

\section{Figures}




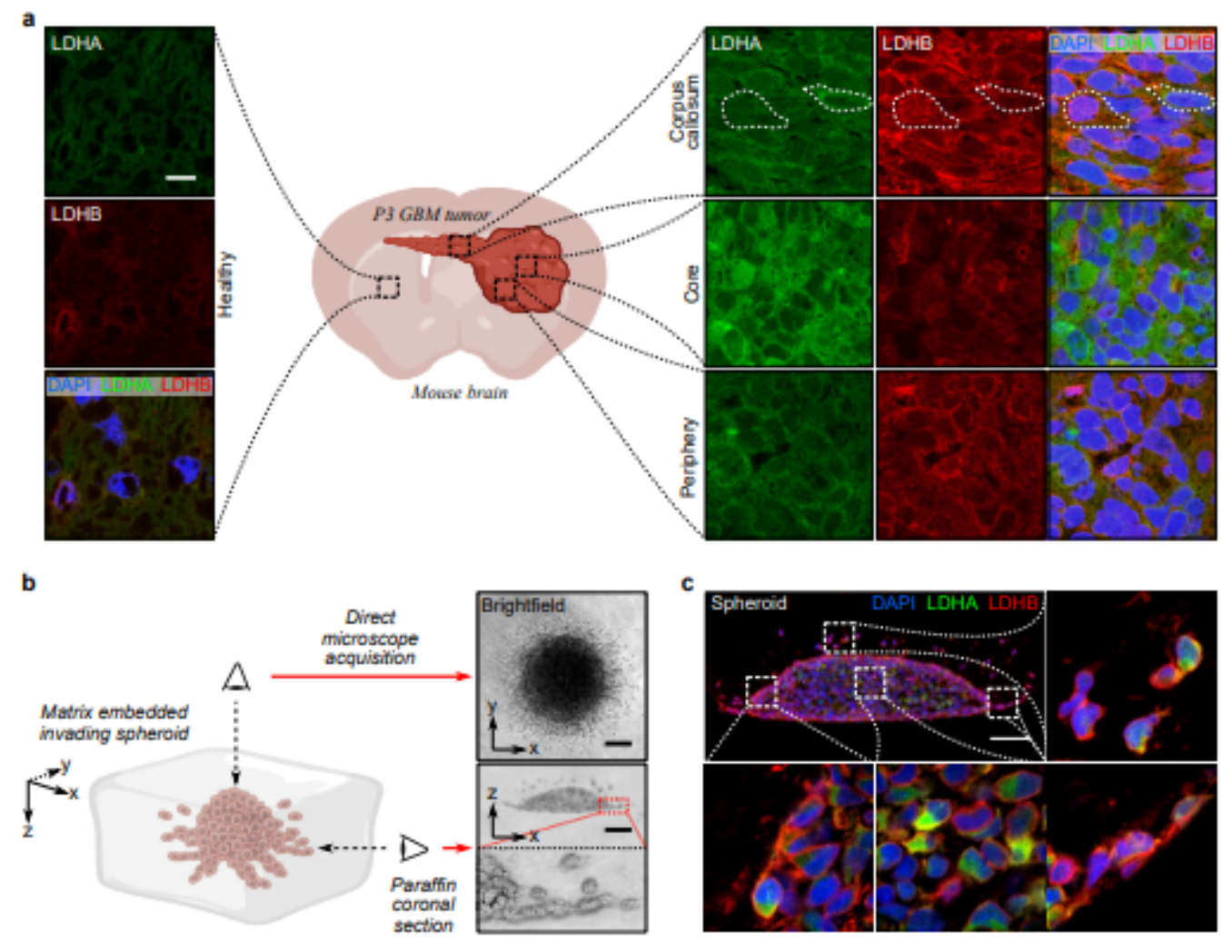

\section{Figure 1}

Distinctive expression of lactate dehydrogenase A and B in GBM. a. Coronal section of P3 spheroids implanted in mouse brain, embedded with paraffin and stained with DAPI (blue), LDHA (green) and LDHB (red). Magnification shows areas as depicted in the central illustration. Scale: $15 \mu \mathrm{m}$. b. Left, 3D representation of an invading P3 spheroid embedded in a collagen matrix. Right, upper view: brightfield microscope image, lower view: coronal section of an invading spheroid. Scale: $100 \mu \mathrm{m}$. c. Coronal section of a P3 spheroid embedded with paraffin and stained with DAPI (blue), LDHA (green) and LDHB (red). Magnification boxes show different areas as depicted in the main image. Scale: $100 \mu \mathrm{m}$. 

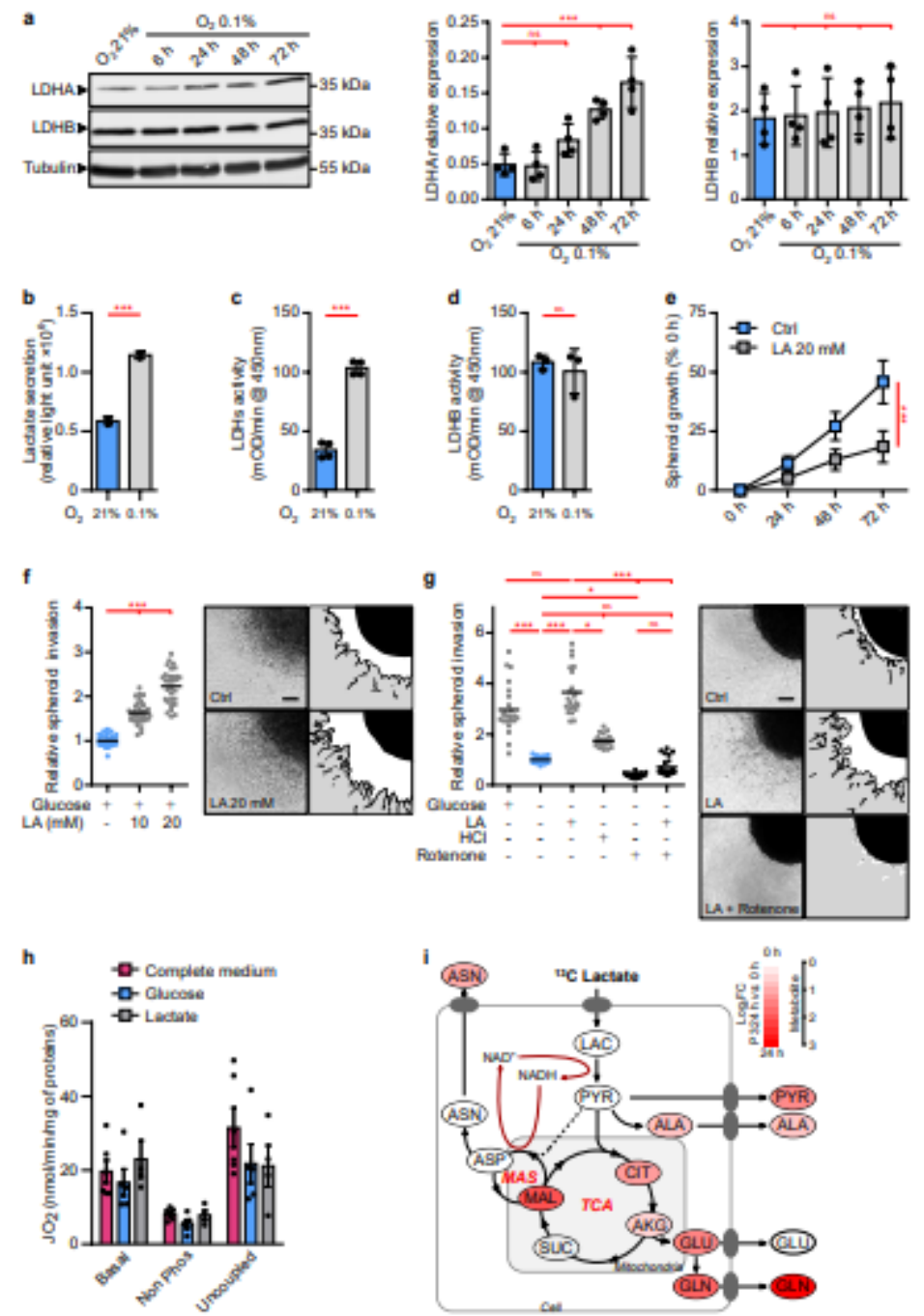

\section{Figure 2}

Increased lactate production by hypoxia, fuels TCA cycle, and drives cell invasion. a. Western blot analysis of LDHA and LDHB from P3 cells upon exposure to $21 \%$ or $0.1 \% 02$ for $6,24,48$ and $72 \mathrm{~h}$. The graphs represent densitometry quantification of immunoblots normalized to tubulin $(n=4)$. b. Lactate secretion of P3 cells exposed to 21 or $0.1 \% 02$ measured with a bioluminescent assay using a proluciferin reductase substrate converted to luciferin in the presence of $\operatorname{NADH}(n=3) . c, d$. Colorimetric assays for the activity of (c) total LDH enzymes $(n=4)$ or $(d)$ immune-captured LDHB enzyme in P3 cells $(\mathrm{n}=3)$. e. P3 spheroid growth recorded over $72 \mathrm{~h}$ during incubation with or without $20 \mathrm{mM}$ lactate. Area of spheroids were measured at $0,24,48$ and $72 \mathrm{~h}$ and growth is represented as a percentage of spheroid area compared to time 0 (8-10 spheroids per condition, $n=3)$. f. P3 spheroid invasion in collagen I gel incubated $24 \mathrm{~h}$ at $21 \% 02$ and incubated with 10 or $20 \mathrm{mM}$ lactate. Invasion rate is expressed as a fold change to the control. (6-8 spheroids per condition, $n=4)$. Images of representative invasive spheroids in each condition. Scale: $100 \mu \mathrm{m}$. g. P3 spheroid invasion in collagen I gel incubated $24 \mathrm{~h}$ at $0.1 \% 02$ and incubated with $20 \mathrm{mM}$ lactate, $20 \mu \mathrm{M}$ rotenone, and $1.5 \mathrm{mM} \mathrm{HCl}$. Invasion rate is expressed as a fold change of the control (6-8 spheroids per condition, $n=4)$. Images of representative invasive spheroids in 
each condition. Scale: $100 \mu \mathrm{m}$. h. P3 cell mass-specific respiration obtained by oxygraphy analysis (left) and inhibitors sensitivity ratio standardized for their respective maximal respiratory capacity (right). Cells were cultured in (1) complete medium or in medium without glucose and pyruvate supplemented with either (2) $10 \mathrm{mM}$ glucose or (3) $20 \mathrm{mM}$ lactate $(\mathrm{n}=4)$. JO2 Basal: baseline respiratory capacity, J02 nonphosphorylating (non phos): minimal respiratory capacity and $\mathrm{JO} 2$ uncoupled: maximal respiratory capacity. i. Metabolic changes of central carbon metabolism on P3 cells infused with [13C]lactate for 24 h. Metabolites are labelled with a colored oval whose color corresponds to log2 fold changes between 24 $\mathrm{h}$ and $\mathrm{O}$ h (red, increase in $24 \mathrm{~h}$ ). TCA, TriCarboxylic Acid cycle; MAS, Malate-Aspartate Shuttle; LAC, lactate; PYR, pyruvate; CIT, citrate; AKG, alpha-ketoglutarate; SUC, succinate; MAL, malate; ALA, alanine; GLU, glutamate; GLN, glutamine; ASP, aspartate; ASN, asparagine. See also Suppl Figure 3. Data are represented as mean $\pm s . d(a, b, c, d, e)$, as mean $(f, g)$ or as mean \pm s.e.m (h). $n$ represents independent experiments. P values were calculated using One-Way ANOVA following by Dunnett's multiple comparisons test (a), unpaired t test (b, c, d, e), Kruskal-Wallis test following by Dunn's multiple comparisons test (f, g), Two-way ANOVA following by Tukey's multiple comparisons test $(h)$; ${ }^{*}<0.05$; ${ }^{\star *} \mathrm{P}<0.01 ; * \star * \mathrm{P}<0.001 ; \mathrm{ns}$, not significant. Exact $\mathrm{P}$ values are provided in the Source Data.

a

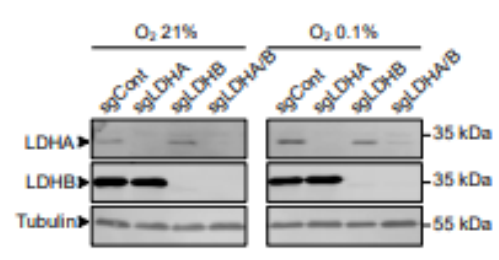

b

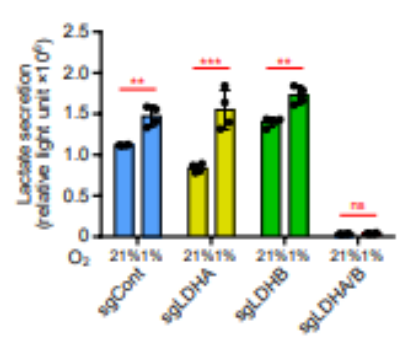

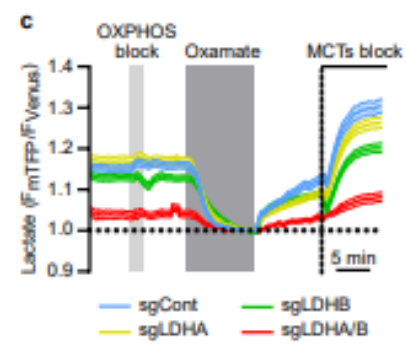
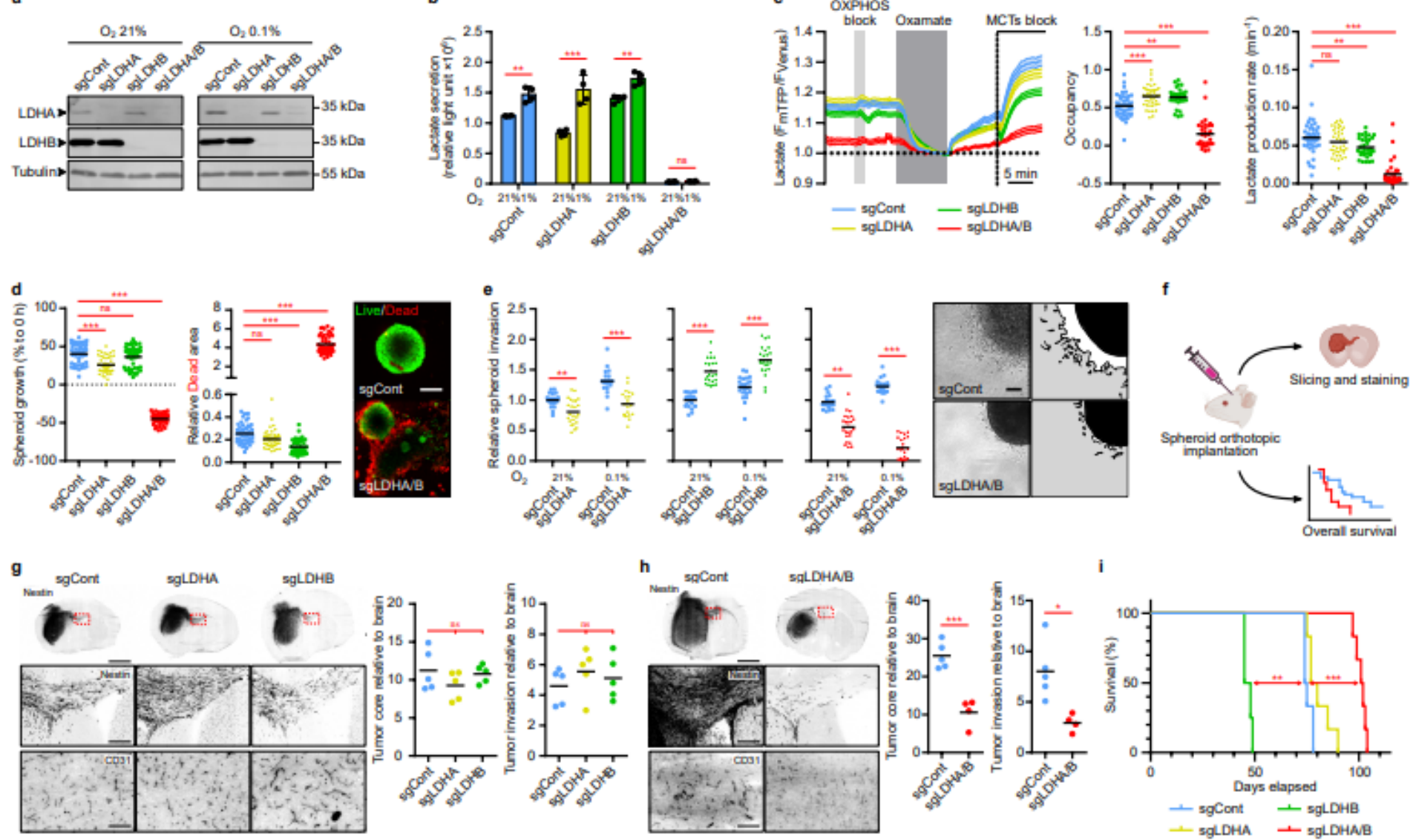

\section{Figure 3}

Double knockout of LDHA and LDHB impairs GBM lactate metabolism, viability, and invasion a. Western blot analysis of LDHA and LDHB expression in P3 cells transduced with Crispr-Cas9 lentiviral vectors with 
scramble sequence (sgControl) or against LDHA (sgLDHA), LDHB (sgLDHB) or both (sgLDHA/B). Knockout (KO) cells were exposed to $21 \%$ or $1 \% 02$ for 48 h. b. Lactate secretion of P3 cells KO for LDHA, LDHB or both, exposed to $21 \%$ or $1 \%(n=3)$. c. Intracellular lactate level analyzed with a fluorescent biosensor in P3 cells (control, KO for LDHA, LDHB or both). Left, lactate level monitored in basal condition, followed by sequential exposure to OXPHOS block ( $5 \mathrm{mM}$ sodium azide), $6 \mathrm{mM}$ oxamate and MCTs blockers ( $1 \mu \mathrm{M}$ AR-C1558585 + 1 mM diclofenac). The response to oxamate and MCTs blockers was used to determine, Middle, the basal lactate level (expressed as biosensor occupancy) and, Right, the lactate production rate ( $n=4,33-44$ cells analyzed per condition). See material and methods for analysis details and see also Supplementary Figure 4. d. Left: P3 spheroid growth recorded over one week at $1 \%$ 02 (8-10 spheroids per condition, $\mathrm{n}=3$ ). Right: Viability of spheroids at one week, incubated with calcein (green) or ethidium homodimer-1 (red). Scale: $200 \mu \mathrm{m}$. e. P3 spheroid invasion in collagen I gel, incubated $24 \mathrm{~h}$ at $21 \%$ or $0.1 \%$ 02. Invasion rate is expressed as fold change from controls (6-8 spheroids per condition, $n=4$ ). Images of representative of control or LDHA/B KO spheroids. Scale: $100 \mu \mathrm{m}$. $\mathrm{f}$. Schematic representation of the orthotopically xenotransplanted P3 spheroids into Ragy2 C-/- mice. A first cohort was sacrificed when one mouse reached a limit point, brains was extract, snap frozen in liquid nitrogen, sliced and stained. For the second cohort, each mouse was sacrificed when it reached a limit point allowing the draw of survival curves. g-h. Left: Immunofluorescence staining of Nestin (top and middle) and CD31 (bottom) of control and LDHA/B KO P3 tumor section. Scales: 2 mm (top), $250 \mu \mathrm{m}$ (middle) and $100 \mu \mathrm{m}$ (bottom). Right: Graphs represent tumor core and invasion area of control and LDHA/B KO P3 tumors (average of 5-6 sections per tumor, $n=5$ mice per group). i. Kaplan-Meier survival curves of xenotransplanted mice with P3 cells KO for LDHA (yellow), LDHB (green), LDHA/B (red) or control (blue) ( $n=8$ mice per group). Data are represented as mean \pm s.d (b), as mean \pm s.e.m (c - left part) or as mean (c, d, e, g, h). $n$ represents independent experiments or biological replicates. $P$ values were calculated using Two-way ANOVA following by Tukey's multiple comparisons test (b, e), KruskalWallis test following by Dunn's multiple comparisons test $(c, d)$, One-Way ANOVA following by Dunnett's multiple comparisons test $(\mathrm{g})$, unpaired $t$ test $(\mathrm{h})$ or Log-rank (Mantel-Cox) test (i); ${ }^{*} \mathrm{P}<0.05$; ${ }^{* *} \mathrm{P}<0.01$; ${ }^{* *} \mathrm{P}<0.001$; ns, not significant; exact $\mathrm{P}$ values are provided in the Source Data. 

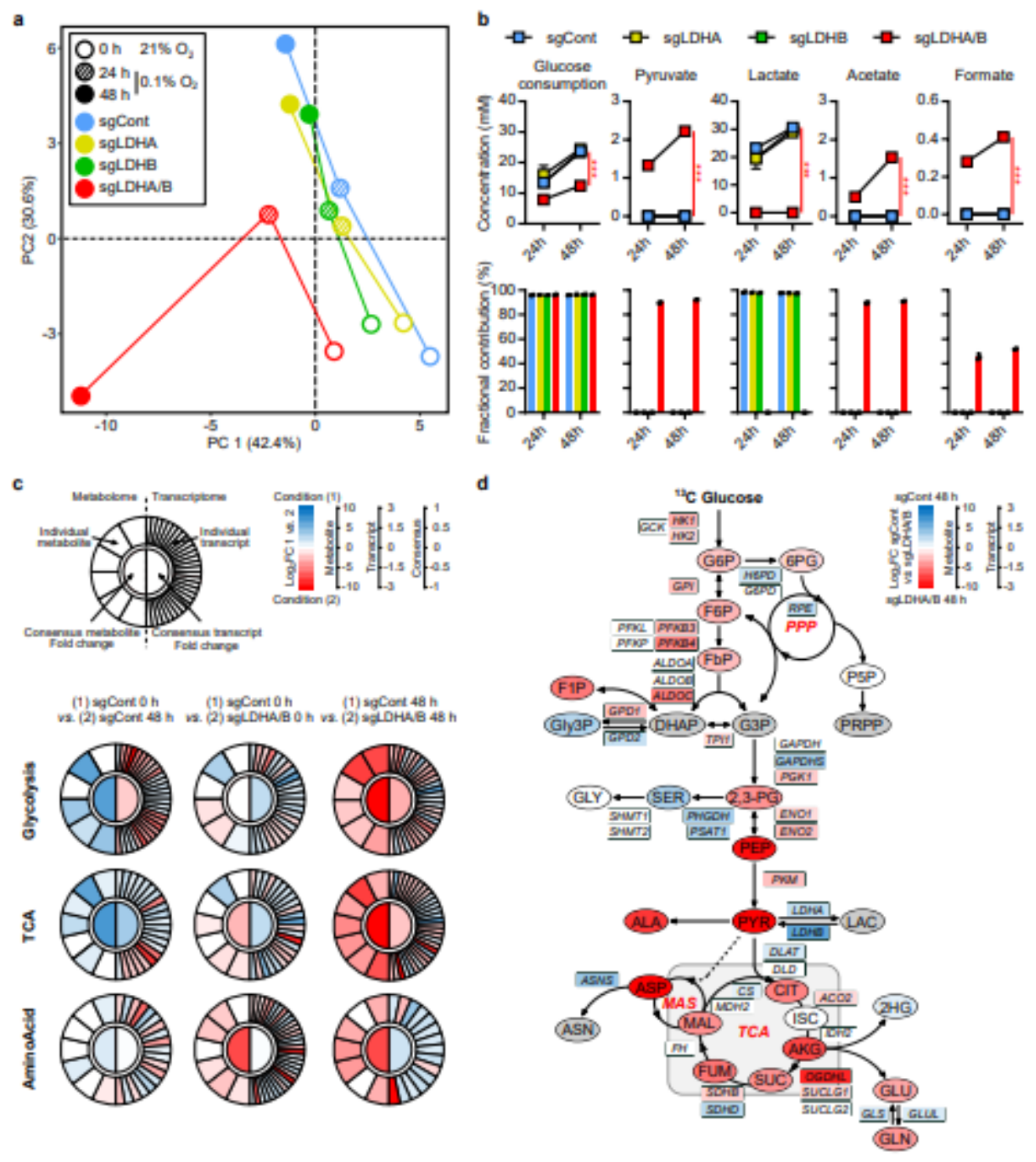

\section{Figure 4}

Correlation between transcriptomic and metabolomic analysis reveals dysregulated metabolic pathways in double knock out LDHA/B. LDH KO P3 cells were infused 0, 24 and $48 \mathrm{~h}$ with [13C]glucose at 1\% 02. a. Principal component analysis of metabolomic data from cell extracts. b. Metabolites from cell medium. Top, concentration of metabolite consumed ([13C]glucose) or secreted (pyruvate, lactate, acetate, formate) by cells. Bottom, amount of labeled isotopes relative to the total amount of this element, expressed as a percentage (fractional contribution). c. Circular metabologram illustrating metabolic and transcriptomic differences in metabolite pathways between LDH KO P3 cells. The metabologram is divided in two parts, the left corresponds to metabolomic analysis and the right to the transcriptomic analysis. The outer circle corresponds to the log2 fold change for each metabolite (left) and transcripts (right). The central circle displays the average fold change of all analytes. $d$. Metabolic changes of central 13-labeled-carbon metabolism in LDH KO P3 cells infused with [13C]glucose at $1 \% 02$ for $48 \mathrm{~h}$. Metabolites are labelled with a colored-oval and enzyme transcripts with a colored-square, whose color corresponds to the log2 fold changes between sgControl and sgLDHA/B (blue, increase in sgControl; red, increase in sgLDHA/B; gray, not measured or not computable). For legend, see also Figure 2i; PPP: 
Pentose Phosphate Pathway; G6P: Glucose-6-Phosphate; 6PG: 6-Phosphogluconate; F6P: Fructose-6Phosphate; FbP: Fructose-bisPhosphate; P5P: Pyridoxal-5-Phosphate; F1P: Fructose-1-Phosphate; Gly3P: Glycerol-3-Phosphate; DHAP: Dihydroxyacetone Phosphate; G3P: Glyceraldehyde-3-Phosphate; PRPP: Phosphoribosylpyrophosphate; GLY: Glycine, SER: Serine; 2,3-PG: 2,3-Phosphoglycerate; PEP:

Phosphoenolpyruvate; 2HG: 2-Hydroxyglutarate. See also Suppl Figure 5.
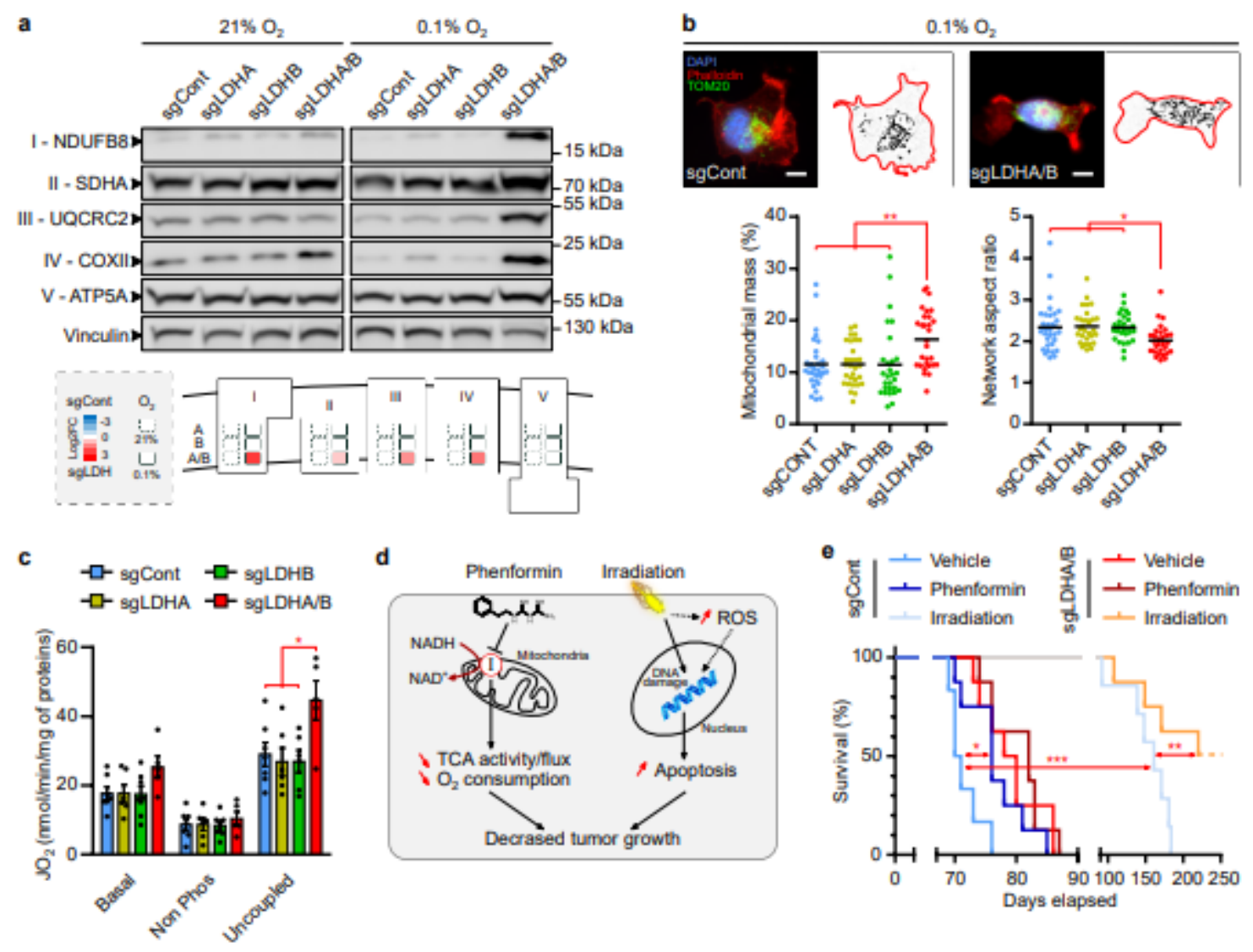

\section{Figure 5}

Knock out LDHA/B affect mitochondrial functions and sensitize tumors to radiation. a. Western blot analysis of mitochondrial respiratory chain subunits from LDH KO P3 cells upon exposure to $21 \%$ or $0.1 \%$ 02. Roman numbers indicate the respiratory complex number. The diagram below represents densitometry quantification of the immunoblots normalized to vinculin and expressed as log2 foldchange to control cells in $21 \%$ and $0.1 \% 02(n=3)$. b. Epifluorescence (top) and quantitative image analysis (bottom) of immune stained mitochondria (TOM20, green) from LDH KO P3 cells (Phalloidin, red; DAPI, blue) upon exposure to $0.1 \% 02$ (28-32 cells per group). Mitochondrial mass corresponds to the area covered by the mitochondria relative to the entire cell area. Network aspect ratio is the ratio of the major axis to minor axis of the mitochondrial network. Scale: $10 \mu \mathrm{m}$. c. LDH KO P3 cell mass-specific respiration obtained by oxygraphy analysis $(n=4)$. For details, see Figure $2 \mathrm{~h}$. d. Schematic representation of phenformin or irradiation effects on tumor cells. e. Kaplan-Meier survival curves of xenotransplanted mice with LDHA/B KO (red) or control (blue) P3 cells, treated with phenformin or irradiated with $10 \mathrm{~Gy}$. Data are represented as mean (b) or as mean \pm s.e.m (c). $n$ represents independent experiments or 
biological replicates. P values were calculated using Two-way ANOVA following by Tukey's multiple comparisons test $(\mathrm{a}, \mathrm{c})$, One-way ANOVA following by Tukey's multiple comparisons test (b) or Log-rank (Mantel-Cox) test (e); ${ }^{*}<<0.05 ; * * P<0.01 ; * \star * P<0.001 ; n s$, not significant; exact $\mathrm{P}$ values are provided in the Source Data.
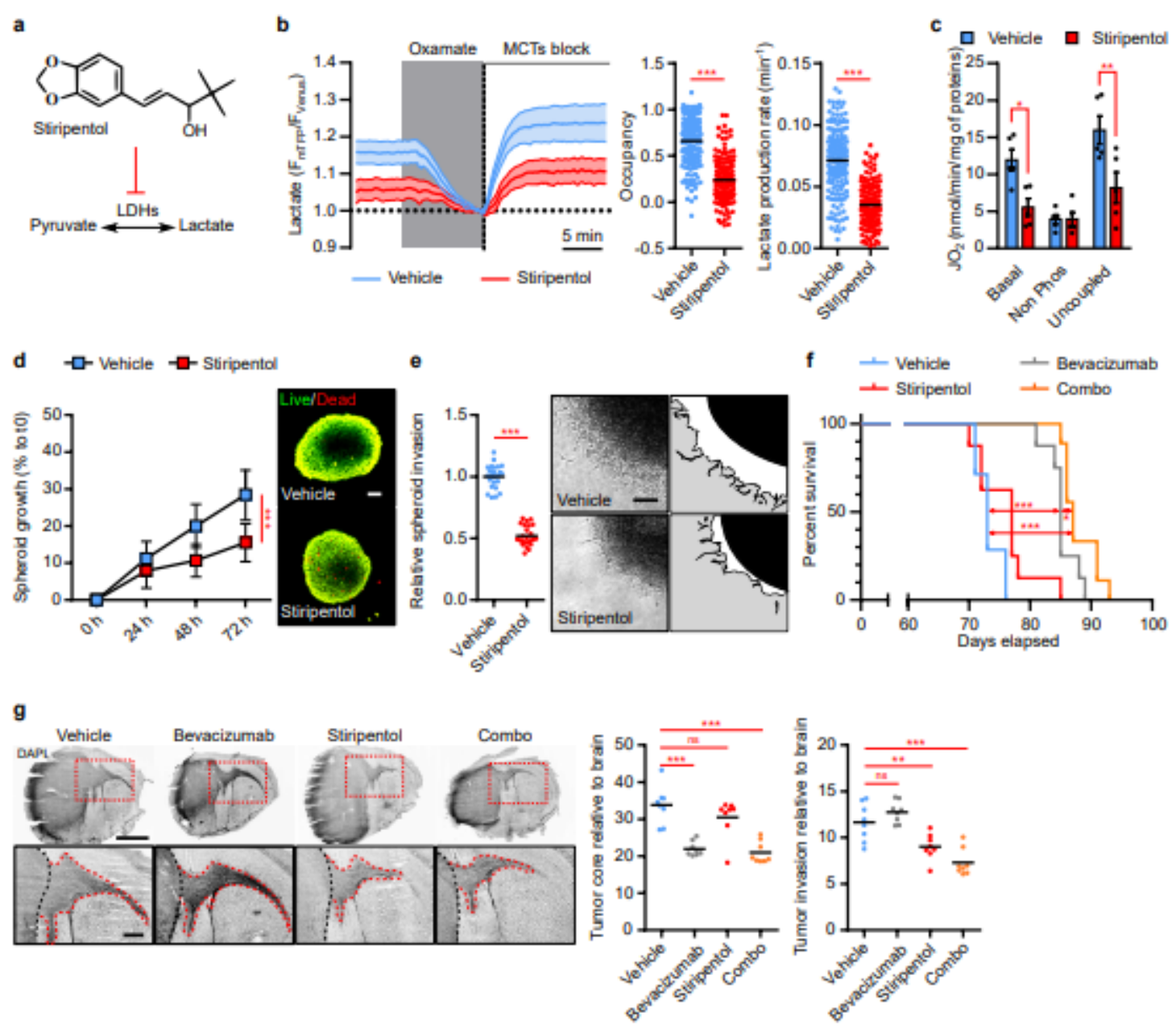

Figure 6

Combination of antiepileptic and antiangiogenic drugs reduce GBM growth a. Stiripentol, an aromatic allylic alcohol drug that inhibits LDH. b. Intracellular lactate level analyzed with a fluorescent biosensor in P3 cells treated with vehicle or $500 \mu \mathrm{M}$ stiripentol. Left, lactate level monitored in basal condition, followed by sequential exposure to $6 \mathrm{mM}$ oxamate and MCTs block $(1 \mu \mathrm{M}$ AR-C $1558585+1 \mathrm{mM}$ diclofenac). The response to oxamate and MTCTs block was used to determine, Middle, the basal lactate level (expressed as biosensor occupancy) and, Right, the lactate production rate ( $n=4,33-45$ cells analyzed per condition). c. P3 cell mass-specific respiration in cells treated with $500 \mu \mathrm{M}$ stiripentol, obtained by oxygraphy analysis $(n=4)$. For details, see Figure $2 \mathrm{~h}$. d. Left: P3 spheroid growth recorded over $72 \mathrm{~h}$ during incubation with stiripentol at $0.1 \% 02$ (8-10 spheroids per condition, $\mathrm{n}=3$ ). Right: Viability of spheroids at $72 \mathrm{~h}$, incubated with calcein (green) or ethidium homodimer-1 (red). Scale: 100 $\mu \mathrm{m}$. e. Left: P3 spheroid invasion in collagen I gel, incubated $24 \mathrm{~h}$ with $500 \mu \mathrm{M}$ stiripentol at $0.1 \% 02$. Invasion rate is expressed as fold change from control (6-8 spheroids per condition, $n=3)$. Right: 
Representative images of invasive spheroids for each condition. Scale: $100 \mu \mathrm{m}$. f. Kaplan-Meier survival curves of xenotransplanted mice with P3 cells. Mice were treated either with vehicle (blue), bevacizumab (gray), stiripentol (red) or both drugs (orange) ( $n=8$ mice per group). g. Left: Immunofluorescence staining of DAPI of treated P3 tumor section. Scales: $500 \mu \mathrm{m}$. Right: The graphs represent the tumor core and invasion area of double LDH KO P3 tumor (average of 5-6 sections per tumor, $n=4-5$ mice per group).

\section{Supplementary Files}

This is a list of supplementary files associated with this preprint. Click to download.

- SourceDataFig4.xlsx

- SourceDataFig3.xIsx

- SourceDataFig5.xlsx

- SourceDataSuppFig2.xIsx

- UncropedWBLDHsHypo.pdf

- SuppFig5210702.pdf

- SuppFig7210608.pdf

- SuppMatMetJune2021DoubleBlind.docx

- SuppFig1210702.pdf

- SuppFig6210608.pdf

- ReagentsList.xlsx

- SuppFig4210608.pdf

- SuppFig2210608.pdf

- SourceDataRNAseq.xIsx

- SuppFig3210608.pdf

- UncropedWBRespChain.pdf

- SourceDataFig6.xIsx

- SourceDataSuppFig4.xlsx

- SourceDataFig2.xlsx

- UncropedWBCRISPRLDHs.pdf 